
711-716

\title{
Early water intake restriction to prevent inappropriate antidiuretic hormone secretion following transsphenoidal surgery: low BMI predicts postoperative SIADH
}

\author{
Junko Matsuyama ${ }^{1,2}$, Hidetoshi lkeda', Shunsuke Sato', Koh Yamamoto', \\ Genichiro Ohashi ${ }^{1}$ and Kazuo Watanabe ${ }^{1}$ \\ ${ }^{1}$ Research Institute of Pituitary Disease, Southern Tohoku General Hospital, Koriyama, Fukushima, Japan and \\ ${ }^{2}$ Department of Neurosurgery, Southern Tohoku General Hospital, 1-2-5, Satonomori, Iwanuma, Miyagi 989-2483, \\ Japan
}

Correspondence should be addressed

to J Matsuyama

Email

junko9106@hotmail.com

\begin{abstract}
Objective: The goals of this study were to assess the incidence of and risk factors for the syndrome of inappropriate antidiuretic hormone secretion (SIADH) in patients following transsphenoidal surgery (TSS), and to validate the effectiveness of early prophylactic restriction of water intake.

Design: Retrospective analysis was performed for 207 patients who had undergone TSS, including 156 patients not placed on early prophylactic water restriction. Sixty-four patients received treatment for SIADH.

Methods: We compared the incidence of SIADH between patients with and without early water intake restriction, and analyzed various risk factors for SIADH using statistical analyses.

Results: BMI was significantly lower for patients with SIADH than for those patients without SIADH. Statistical analysis revealed that the threshold BMI predicting SIADH was 26. Serum sodium levels on postoperative days 5-10 and daily urine volumes on postoperative days 5-10 were significantly lower in patients with SIADH than in those without SIADH. Postoperative body weight loss on days $6,8,10$, and 11 was significantly higher in patients with SIADH. The incidence of SIADH after starting prophylactic water intake restriction (14\%) was significantly lower than the rate before early water restriction (38\%; $P<0.05)$.

Conclusions: SIADH is relatively common after TSS, and serum sodium concentrations and daily urine volumes should be carefully monitored. Patients with low preoperative BMI should be closely observed, as this represented a significant preoperative risk factor for SIADH. Early prophylactic water intake restriction appears effective at preventing postoperative SIADH.
\end{abstract}

\section{Introduction}

Disturbances in fluid and sodium balances are commonly encountered in neurosurgical patients $(1,2,3,4)$. Following transsphenoidal surgery (TSS) for pituitary tumors and other sellar tumors, patients are at high risk for developing water balance disorders, including diabetes insipidus (DI) and syndrome of inappropriate antidiuretic hormone secretion (SIADH). The goals of this study were to assess the incidence of and risk factors for SIADH in patients who had undergone TSS, and to validate the effectiveness of early prophylactic water intake restriction. Postsurgical hyponatremia typically occurs in a delayed fashion, and there is currently no established standard of care for the management of delayed hyponatremia due to SIADH after TSS. (c) 2014 European Society of Endocrinology Printed in Great Britain 
We clarified the potential risk factors for SIADH after TSS, and performed a unique assessment of early prophylactic water intake restriction.

\section{Subjects and methods}

Between January 2012 and July 2013, a total of 207 patients (129 females and 78 males) underwent TSS in the Research Institute for Pituitary Disease at Southern Tohoku General Hospital, Japan. All patients provided informed consent for participation in the study. Institutional Review Board approval was received for all study protocols. Mean patient age was $44.6 \pm 18.1$ years (range, $9-82$ years).

SIADH was diagnosed based on the following criteria: serum sodium $<135 \mathrm{mEq} / \mathrm{l}$; no dehydration; normal renal function (serum creatinine $<1.2 \mathrm{mg} / \mathrm{dl}$ ); low effective serum osmolality $(<275 \mathrm{mOsm} / \mathrm{l})$; and high urinary sodium. The absence of dehydration is the key characteristic that distinguishes SIADH from cerebral salt wasting syndrome. We also determined the incidence of and risk factors for postoperative SIADH.

Delayed hyponatremia was defined as a serum sodium concentration $<135 \mathrm{mEq} / \mathrm{l}$ after day 3 . Clinical and radiological features, tumor size, endocrinological activity, hyponatremic symptoms, daily postoperative urine volume, BMI, and postoperative serum sodium levels were investigated. BMI was calculated based on the height and body weight of each patient. Postoperative serum sodium levels were measured on day 1 and days 3-11.

We compared the BMI of the patients with and without SIADH. We also compared postoperative daily serum sodium levels and daily urine volumes. We then attempted to determine the threshold BMI predicting postoperative SIADH.

Our study was unique in its management of early prophylactic water restriction. Thus, after postoperative day 6 , we considered the following symptoms: i) serum sodium level $<140 \mathrm{mEq} / \mathrm{l}$; ii) daily urine volume $<1000 \mathrm{ml}$, and iii) body weight gain (compared with preoperative body weight).

If two or more of the three above conditions were met, early restriction of water intake was initiated $(<200 \mathrm{ml}$ of extra water beyond the $\sim 1600 \mathrm{ml}$ present in daily meals for hospitalized patients). Steroid replacement tapering was routinely performed after TSS from days 1 to 12 . Rathke's cleft cyst was analyzed separately from other pituitary adenomas.

For statistical analysis, we used Student's t-test, the Mann-Whitney $U$ test, the $\chi^{2}$ test, Fisher's exact probability-test, and receiver operating characteristic (ROC) curve analysis with a 95\% CI. The statistical significance was defined as a probability value $<0.05$. Data analysis was performed using StatFlex version 6.0 Software (Artec, Osaka, Japan).

\section{Results}

In total, 64 of the 207 patients presented with SIADH (delayed hyponatremia). Only one patient showed symptoms of SIADH (nausea and vomiting).

Among the patients who underwent TSS, the underlying pathology was Rathke's cleft cysts in 112 patients, adrenocorticotropic hormone (ACTH)-secreting adenomas in 17 patients, growth hormone (GH)-secreting adenomas in 20 patients, plurihormonal adenomas in 25 patients, nonfunctioning adenomas in 21 patients, gonadotroph adenomas in 15 patients, and prolactinomas in eight patients. As the eight prolactinomas were dopamineresistant, all these patients underwent surgery.

Among the 64 patients with SIADH, serum sodium levels began to decrease on postoperative day 5 on average (occurrence of postoperative SIADH), with the nadir occurring on day 9.

We began prescribing early prophylactic water intake restriction in April 2013 whenever a patient fulfilled two or more of the criteria described in the 'Subjects and methods' section. Although the definition of serum sodium levels in SIADH is $<135 \mathrm{mEq} / \mathrm{l}$, we began this prophylactic restriction at an early stage.

Before instituting early prophylactic restriction of water intake, 143 patients underwent TSS at our institution, of whom 55 (38\%) presented with SIADH. After beginning early water intake restriction, 64 patients underwent TSS, of whom nine showed SIADH (14.0\%). The $\chi^{2}$ test revealed that the incidence of SIADH was significantly lower in the early water restriction group than in the group before early water intake restriction $(P=0.00013)$. As Rathke's cleft cyst may have a higher incidence of SIADH compared with other pituitary tumors, we performed separate analyses. Among the 112 cases of Rathke's cleft cyst, the incidence of SIADH before the implementation of early water restriction was 37 in 73 cases (50.7\%), compared with five among 39 cases (12.9\%) after starting early water restriction. The incidence of SIADH among patients with Rathke's cleft cyst was thus significantly decreased with the implementation of water restriction $(P=0.0008)$.

The incidence of SIADH in patients with pituitary adenoma before early water restriction was $25.7 \%$ (18 of 70 cases), compared with $16.0 \%$ (four of 25 cases) after starting early water restriction. Although no significant 
difference was apparent $(P=0.3)$, the incidence of SIADH clearly showed a decreasing tendency after starting early water restriction in patients with pituitary adenoma.

We then examined risk factors for postoperative SIADH. The preoperative BMI of each patient was calculated based on body weight and height. The mean ( \pm s.D.) BMI of patients with SIADH was $21.82 \pm 2.78 \mathrm{~kg} / \mathrm{m}^{2}$, whereas the mean BMI of patients without SIADH was $24.95 \pm 5.19 \mathrm{~kg} / \mathrm{m}^{2}$. Both Student's $t$-test and the MannWhitney $U$ test revealed that BMI was significantly lower in patients with SIADH than in patients without SIADH $(P=0.00012)$. Using ROC curve analysis, we determined the cut-off BMI most predictive of SIADH. BMI correlated with the frequency of SIADH with high specificity and sensitivity at a threshold of $26 \mathrm{~kg} / \mathrm{m}^{2}$. Thresholds above 26 showed lower sensitivity, while specificity was too low for thresholds below $26 \mathrm{~kg} / \mathrm{m}^{2}$. BMI $<26 \mathrm{~kg} / \mathrm{m}^{2}$ might offer a predictor of the occurrence of postoperative SIADH (Fig. 1).

Next, we evaluated the relationships between the incidence (frequency) of SIADH and tumor size and extension. Comparison of the incidence of SIADH between tumors with and without extrasellar extension by $\chi^{2}$ test showed no significant difference $(P$ value $=$ 0.067). Tumor size did not affect the frequency of SIADH in our series $\left(P=3.35, \chi^{2}\right.$ test, stratified based on tumor $>10 \mathrm{~mm}$ in diameter vs tumor $\leq 10 \mathrm{~mm}$ ). We also analyzed correlations between the incidence of SIADH and endocrinological functions. The incidence of SIADH in patients with Rathke's cleft cyst was 37\%, while the incidence of SIADH in patients with GH-secreting adenomas was $7.7 \%$, and the $\chi^{2}$ test revealed this as a significant difference. The incidence of SIADH in patients with ACTHsecreting adenoma was also $7.7 \%$, significantly lower than that in patients with Rathke's cleft cyst. However, we were unable to identify any significant difference in the incidence (frequency) of SIADH among patients with pituitary adenomas, whether plurihormonal adenomas, GH-secreting adenomas, ACTH-secreting adenomas, or prolactinomas. Endocrine activity thus did not correlate with the incidence of SIADH.

The mean age of patients with SIADH was $44.07 \pm$ 18.08 years (mean \pm s.D.), whereas $44.98 \pm 18.07$ (mean \pm S.D.) years in patients without SIADH. No significant difference in age was seen between these two groups $(P=0.79)$. No significant difference was observed between females and males in the incidence of SIADH, either $(P=0.98)$.

We measured serum sodium levels in each patient who underwent TSS on postoperative day 1 , and on days $3-11$. First, mean serum sodium levels in patients with and

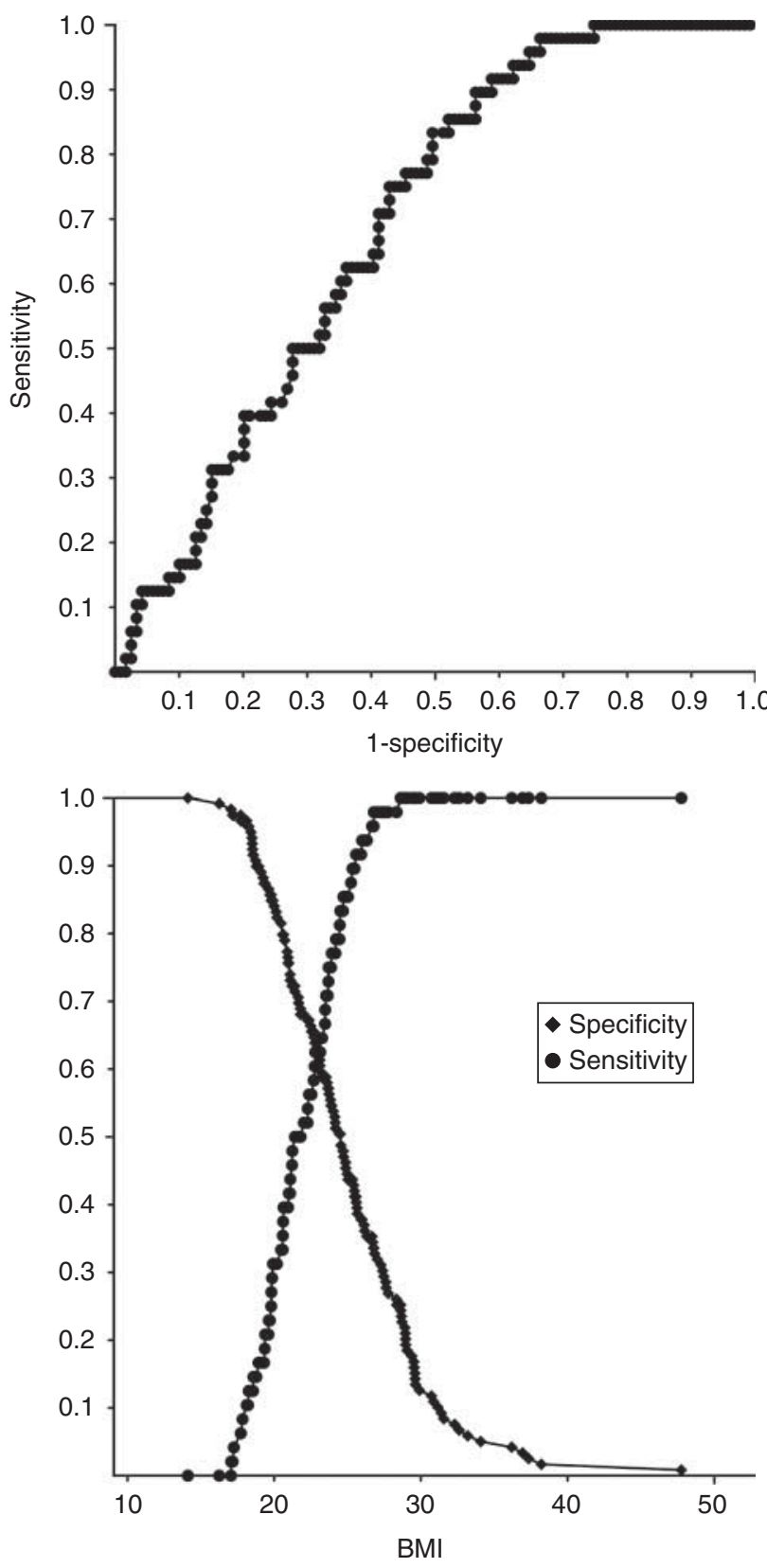

Figure 1

ROC curve analysis revealed that the threshold $B M I$ value predicting SIADH was 26 .

without SIADH were compared on each day. Mean serum sodium levels in patients with SIADH on postoperative days 5-10 were significantly lower than those in patients without SIADH, particularly on days 8 and 9. Serum sodium levels began to decrease on postoperative day 5 (occurrence of postoperative SIADH), with the nadir occurring on day 9 (Fig. 2). The mean serum sodium level in patients with SIADH on day 9 was 

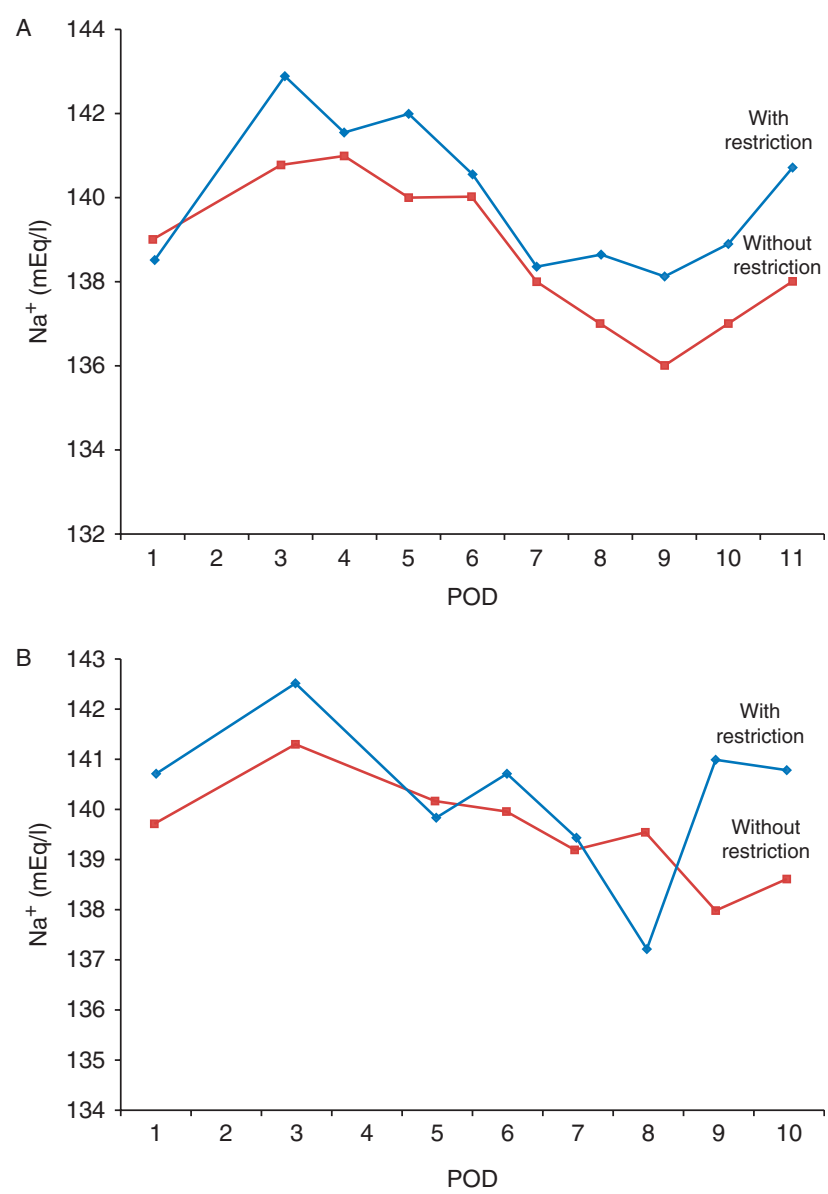

Figure 2

Serum sodium levels with and without water intake restriction in patients with Rathke's cleft cyst (A) and those with other pituitary tumors (B). The nadir of serum sodium level occurred on postoperative day 9. Among the patients with Rathke's cleft cyst, mean serum sodium levels on postoperative days 8-10 were significantly higher in patients with early water restriction than in patients without water intake restriction $(P<0.05)$.

$132.8 \pm 2.64 \mathrm{mEq} / \mathrm{l}$, whereas the mean serum sodium level in patients without SIADH on the same day was $138.57 \pm$ $2.28 \mathrm{mEq} / 1$. The difference between these groups was most pronounced on day 9. Secondly, mean serum sodium levels on each day were evaluated in patients with and without prophylactic water restriction, with separate evaluations of patients with Rathke's cleft cyst and those with other pituitary tumors (Fig. 2A and B). Among the patients with Rathke's cleft cyst, mean serum sodium levels were significantly higher in patients with early water restriction on postoperative days 8-10 than those in patients without water intake restriction (Fig. 2A; $P<0.05)$. In patients with other pituitary tumors, no significant differences in serum sodium levels were observed between with and without water intake restriction (Fig. 2B).

The daily urine volume of each patient was measured from days 1 to 11 . Urine volume began to decrease on day 5 , and daily urine volumes on days 5-10 were significantly lower in patients with SIADH than in those without SIADH. The 24-h volume of excreted urine was evaluated separately in patients with Rathke's cleft cyst and other pituitary tumors (Fig. 3A and B). Mean daily urine volumes on days 7-10 were significantly lower in patients with water restriction than in those without water restriction, both among patients with Rathke's cleft cyst (Fig. 3A) and among those with other pituitary tumors (Fig. 3B) $(P<0.05)$.

The extent of postoperative body weight reduction on days $6,8,10$, and 11 was significantly lower in patients without SIADH compared with patients with SIADH.
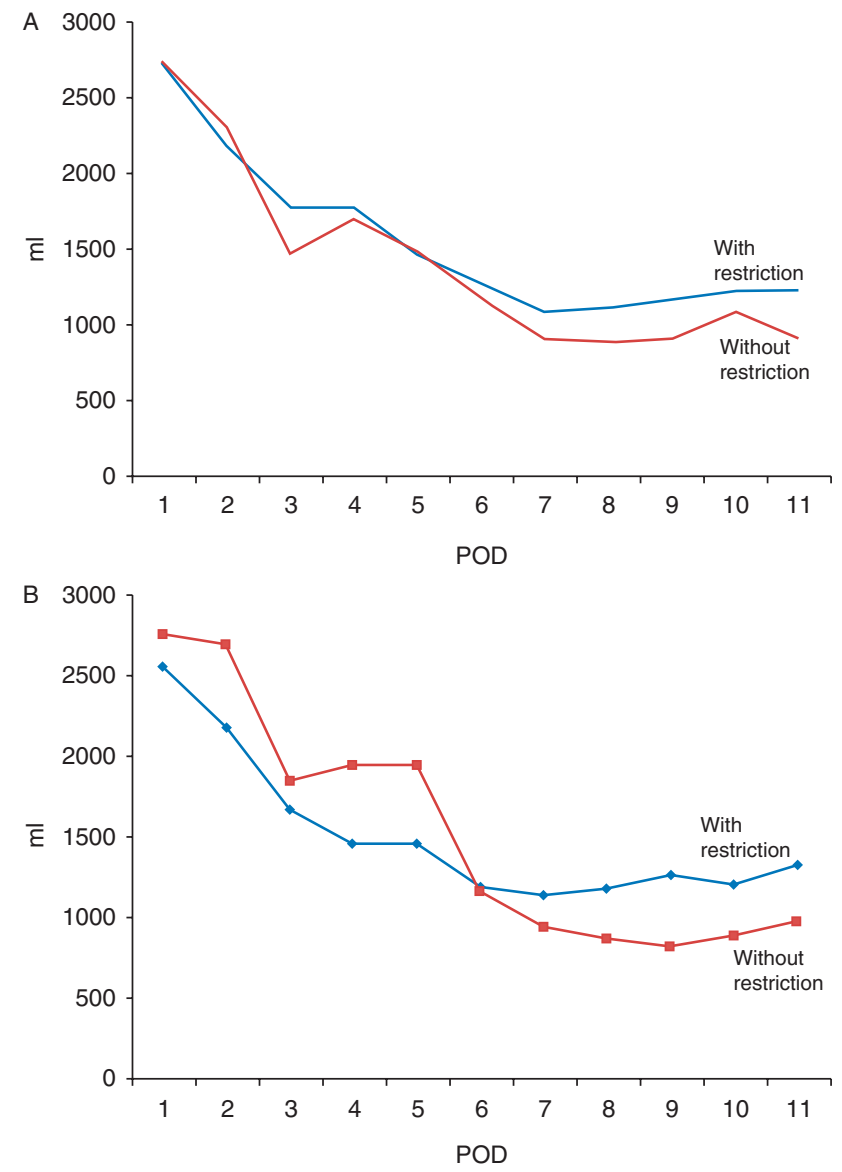

Figure 3

Daily urine volumes with and without water restriction in patients with Rathke's cleft cyst (A) and those with other pituitary tumors (B). Nadir urine volumes occurred on postoperative days 8 and 9 . 


\section{Discussion}

The results of this study indicate that SIADH is relatively more common among patients who undergo TSS than that previously reported, with a frequency of between 2.3 and $35 \%(1,2,3,4,5,6)$. However, the precise incidence of delayed hyponatremia after TSS for pituitary region tumors is difficult to ascertain. In our series, SIADH occurred in 64 of 207 patients who underwent TSS, representing a frequency of $30.6 \%$.

The frequency of SIADH in the cohort without water intake restriction was quite high compared with previous reports, which have usually described incidences between 8.5 and $25 \%(2,5,7)$. Various reasons might explain this finding. First, the rate of smaller tumors was higher than in previous reports, so more extensive gland exploration was required to identify and resect the lesions. As discussed below, release of antidiuretic hormone (ADH) stores from neurohypophyseal cells manipulated during such exploration results in natriuresis $(3,4,6)$. A second factor might be that all patients remained hospitalized for a minimum of 12 days after surgery and serum sodium levels were observed as part of the postoperative management protocol. Certain articles have reported that even normonatremic patients exhibit dysfunctional osmotic regulation of arginine vasopressin secretion, so clinical hyponatremia represents only the 'tip of the iceberg' (7).

Several hypotheses for the mechanisms by which SIADH develops after TSS have been proposed. One such mechanism involves the release of ADH stores from manipulated neurohypophyseal cells, resulting in natriuresis and fluid retention. Surgery may acutely relieve the chronic distortion of the neurohypophyseal stalk and hypothalamus or partially injure the neurohypophyseal stalk, resulting in an ADH surge and, ultimately, a state of fluid overload and hyponatremia $(1,2,3,4)$.

Another potential mechanism by which SIADH could develop is glucocorticoid deficiency. Hyponatremia may be the presenting feature of adrenocortical insufficiency, and ADH may be stimulated by ACTH deficiency $(3,4,8)$.

Regarding the risk factors for SIADH after TSS, such as age, sex, tumor size, tumor type, and development of transient DI, have all been discussed previously $(1,2,3,4)$. In our series, no correlation between tumor size and frequency of SIADH was found. Kelly et al. (2) reported that patients with macroadenomas were more prone to develop delayed hyponatremia than patients with smaller tumors, because surgical removal is more likely to disturb the neurohypophyseal stalk and hypothalamus. However, other authors have determined that patients with microadenoma are more likely to develop hyponatremia because of the more extensive gland exploration required to identify and resect the lesions (7). One article reported no correlation between SIADH and tumor size (1). Patients with delayed hyponatremia tend to be older, according to some articles $(2,8)$. However, no significant differences by age were seen in our cohort. Zada et al. (4) reported that female patients more frequently had SIADH; however, in our series no significant difference was observed between two sexes.

In our series, lower preoperative BMI was the most significant predictor of postoperative hyponatremia, with a threshold BMI of $26 \mathrm{~kg} / \mathrm{m}^{2}$. Hussain et al. (1) reported BMI as a significant predictor of both immediate and delayed postoperative hyponatremia. They stated that the physiological basis for the association between lower BMI and delayed postoperative hyponatremia remains unclear, but a strong association between obesity and impaired renal function has been noted. Patients with a higher BMI may be less responsive to elevated vasopressin levels after TSS, and would thus be less likely to develop delayed postoperative hyponatremia. The nadir of hyponatremia occurred on postoperative days 8 and 9 in our series. Previously published studies have also determined that the hyponatremic nadir occurred on postoperative day 9 (5). The timing of delayed postoperative hyponatremia after TSS has generally been consistent, beginning on postoperative day 4-7 and resolving within 2-3 weeks.

In our study, the incidence of SIADH was significantly higher among patients with Rathke's cleft cyst than among those with pituitary adenoma. However, endocrine activity showed no correlation with the frequency of SIADH, although some articles have reported that endocrine-inactive patients had a higher risk of developing hyponatremia, and patients with Rathke's cleft cyst and Cushing's disease had significantly lower postoperative sodium levels compared with other tumor types $(3,4)$. Zada et al. (4) reported that tumor cell type did not predict the frequency of hyponatremia, although tumor type did correlate with the severity of hyponatremia.

As for treatment, most authors have recommended the implementation of fluid intake restriction therapy for patients with serum sodium levels $<130 \mathrm{mEq} / 1(1,2,3,4)$. Rare cases of severe hyponatremia with levels lower than $120 \mathrm{mEq} / \mathrm{l}$ require more aggressive treatment, such as infusion of hypertonic saline, vasopressin antagonists, or urea therapy $(2,4)$.

This study was unique in proposing prophylactic water intake restriction as both a treatment and a preventive 
mechanism. To the best of our knowledge, no prospective observational studies focusing on similar early prophylactic water intake restriction have been published. The data showing a decreasing frequency of SIADH after early fluid intake restriction suggest the effectiveness of prophylactic early water intake restriction. The result of increased serum sodium levels on postoperative days 8-10 after instituting early water restriction in patients with Rathke's cleft cyst also suggests the effectiveness of water restriction.

\section{Conclusion}

Delayed hyponatremia is a relatively common event following the removal of pituitary tumors by TSS, and is certainly more frequent than has been previously reported. Patients with low preoperative BMI should be closely monitored, as this factor was significantly associated with postoperative SIADH. Serum sodium concentrations should be measured in all patients after TSS in the immediate postoperative period and a few times between postoperative days 5 and 11. Early prophylactic water intake restriction contributed significantly for prevention of postoperative SIADH and delayed discharge.

\section{Declaration of interest}

The authors declare that there is no conflict of interest that could be perceived as prejudicing the impartiality of the research reported.

\section{Funding}

This research did not receive any specific grant from any funding agency in the public, commercial or not-for-profit sector.

\section{References}

1 Hussain NS, Piper M, Ludlam WG, Ludlam WH, Fuller CJ \& Mayberg MR. Delayed postoperative hyponatremia after transsphenoidal surgery: prevalence and associated factors. Journal of Neurosurgery 2013119 1453-1460. (doi:10.3171/2013.8.JNS13411)

2 Kelly DF, Laws ER Jr \& Fossett D. Delayed hyponatremia after transsphenoidal surgery for pituitary adenoma. Report of nine cases. Journal of Neurosurgery 199583 363-367. (doi:10.3171/jns.1995.83. 2.0363)

3 Lee JI, Cho WH, Choi BK, Cha SH, Song GS \& Choi CH. Delayed hyponatremia following transsphenoidal surgery for pituitary adenoma. Neurologia edico-Chirurgica 200848 489-494. (doi:10.2176/nmc.48.489)

4 Zada G, Liu CY, Fishback D, Singer PA \& Weiss MH. Recognition and management of delayed hyponatremia following transsphenoidal pituitary surgery. Journal of Neurosurgery 2007106 66-71. (doi:10.3171/jns.2007.106.1.66)

5 Kristof RA, Rother M, Neuloh G \& KlingmÜller D. Incidence, clinical manifestations, and course of water and electrolyte metabolism disturbances following transsphenoidal pituitary adenoma surgery: a prospective observational study. Clinical article. Journal of Neurosurgery 2009 111 555-562. (doi:10.3171/2008.9.JNS08191)

6 Sane T, Rantakari K, Poranen A, Tähtelä R, Välimäki M \& Pelkonen R. Hyponatremia after transspheoidal surgery for pituitary tumors. Journal of Clinical Endocrinology and Metabolism 199479 1395-1398.

7 Olson BR, Gumowski J, Rubino D \& Oldfeld EH. Pathophysiology of hyponatremia after transsphenoidal pituitary surgery. Journal of Neurosurgery 199787 499-507. (doi:10.3171/jns.1997.87.4.0499)

8 Kinoshita Y, Tominaga A, Arita K, Sugiyama K, Hanaya R, Hama S, Sakoguchi T, Usui S \& Kurisu K. Post-operative hyponatremia in patients with pituitary adenoma: post-operative management with a uniform treatment protocol. Endocrine ournal 201158 373-379. (doi:10.1507/endocrj.K10E-352)

Received 27 June 2014

Revised 10 September 2014

Accepted 16 September 2014 